\title{
Tracking of Irregular Graphical Structures for Tissue Deformation Recovery in Minimally Invasive Surgery
}

\author{
Marco Visentini-Scarzanella ${ }^{1}$, Robert Merrifield ${ }^{2}$, Danail Stoyanov ${ }^{2}$, \\ and Guang-Zhong Yang ${ }^{1,2}$ \\ ${ }^{1}$ Royal Society/Wolfson Foundation MIC Laboratory, \\ ${ }^{2}$ Institute of Biomedical Engineering, \\ Imperial College London, London SW7 2AZ, United Kingdom \\ \{marcovs, robert.merrifield, danail.stoyanov\} @imperial.ac.uk \\ g.z.yang@imperial.ac.uk
}

\begin{abstract}
Tissue deformation tracking is an important topic of minimally invasive surgery with applications ranging from intra-operative guidance to augmented reality visualisation. In this paper, we present a technique for visual tracking of irregular structures with an arbitrary degree of connectivity in space. The variational formulation of the proposed method ensures that correlation is maximised between tracked points and their computed new positions while the overall structure shape variation is minimised, thus maintaining spatial coherence of the tracked structure. The proposed method is applied to surgical annotation and tracking in 3D for telementoring and path-planning. The results are validated both on a CT-scanned phantom model and in vivo, showing an average alignment error of $1.79 \mathrm{~mm}( \pm 0.72 \mathrm{~mm})$.
\end{abstract}

\section{Introduction}

Tissue deformation tracking during MIS has been the focus of considerable research efforts with the goal of enhancing the user experience particularly during robotically assisted procedures. For MIS, issues such as limited field-of-view, large-scale tissue deformation and disorientation can be addressed by image mosaicing, virtual motion compensation, ablation guidance [1], imposition of dynamic active constraints and motor channelling [2], all of which hinge on reliable image feature tracking techniques [3]. While these techniques could be used in conjunction with $2 \mathrm{D}$ feature tracking plus stereo matching, a 2D approach to tracking does not reflect the physical structure of the object and is unsuitable for integrating a priori motion information.

In this paper, a structure tracking approach is proposed. It differs from feature or point based tracking methods in that its optimality criteria are global to the structure tracked and it can operate in any dimensional space, in contrast to unconstrained feature tracking where the optimality is local to each feature tracked. While existing methods for structure tracking operate on regular grids in $2 \mathrm{D}$, the proposed method relies on the construction and tracking of networks from sparse point clouds with arbitrary degrees of connectivity in $d$ dimensions. A novel feature correlation function tailored to MIS data is formulated and the proposed method is applied to MIS 3D 
spatio-temporal telestration for path planning and telementoring. The method is evaluated with both a synthetic heart model with known ground-truth 3D geometry and deformation, as well as in vivo data acquired during a Totally Endoscopic Coronary Artery Bypass (TECAB) procedure.

\section{Method}

The proposed method consists of three distinct steps. First, a network is built from a collection of points given the parameters about their dimensionality and connectivity level. Then, as their motion fields are updated, a point-wise correlation is computed for each possible position in the search space for all points in the cloud. Finally, a variational technique is used to maximise correlation between the points and their proposed updated positions whilst minimising the overall network shape variation.

\subsection{Network Building}

Given a sparse collection of points in $d$ dimensions, a connected network can be built given a scoring function and the maximum degree of connectivity $k$ allowed. The maximum degree of connectivity is determined by the highest total number of links a point in the network has, divided by two: for instance, a regular 2D mesh would have a degree of connectivity of 2 . Determining the degree of connectivity required implies a trade-off between strong geometric regularisation constraints for high degrees due to the larger number of dependencies and the inability of fully capturing the characteristics of the underlying structure being represented for low degrees.

To construct a network given a set of points and a degree $k$, the $d$-dimensional space is first partitioned into $2 k$ equal sections. For each space partition and each pair of points that could be connected by a line within the partition under consideration, their $L_{d}$ norm is computed, and the pair separated by the minimum distance is connected.

(a)

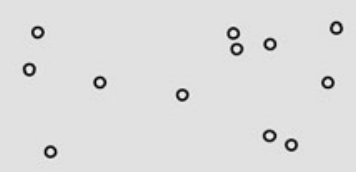

(d)

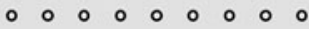

$\begin{array}{lllllllllllllll}0 & 0 & 0 & 0 & 0 & 0 & 0 & 0 & 0\end{array}$

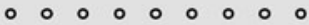

0

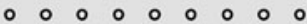

(b)

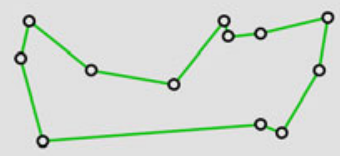

(e)

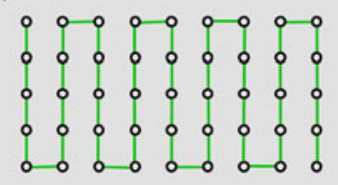

(c)

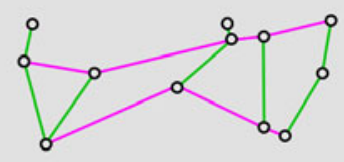

(f)

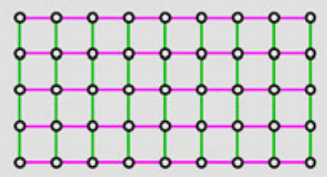

Fig. 1. (a) Sparse set of $2 \mathrm{D}$ points. (b) Result of the algorithm with degree of connectivity $k=1$ and (c) $k=2$. (d) Regular 2D point grid. (e) Result of the algorithm with $k=1$ and (f) $k=2$. 
The process is iterated until no more points can be linked without breaking the limit of two connections for every $k$. For regular grids and $k=d$, the process converges towards a conventional lattice structure, as shown in Fig. 1 above for $d=2$ :

\subsection{Correlation Estimation}

Variational approaches to optical flow estimation commonly involve solving the following minimisation problem [4]:

$$
\min _{u}\left\{\int_{\Omega} \lambda g(x, u(x))+\psi(\nabla u(x)) d \Omega\right\}
$$

In the expression above, $g(\mathbf{x})$ is the data term measuring the correlation between the point $\mathbf{x}$ and the displaced candidate $\mathbf{x}+u(\mathbf{x})$, the second term is a regularisation constraint enforcing a smooth displacement field, and $\lambda$ is a factor weighing the relative contribution of data and regularisation terms.

The nature of the data term depends on the optimisation procedure adopted: faster techniques involve the use of differentiable data terms [5], whereas more sophisticated non-differentiable penalty functions are coupled with complete searches [6]. Recently, Giannarou et al. [7] proposed an affine-invariant anisotropic corner detector tailored for MIS applications and robust to lighting variations and image blur. Detected features are described as ellipses whose size, orientation and radii ratio summarise the structural information of the feature patch examined.

In this study, a non-differentiable penalty function based on the intersection area between the ellipses representing the original and candidate points is used. The measure is then weighed by the difference in colour intensity distribution between the two patches, in order to include both structural and intensity information:

$$
g(\mathbf{x}, u(\mathbf{x}))=1-\varphi\left(I(\mathbf{x}), I^{\prime}(\mathbf{x}+u(\mathbf{x}))\right) \cdot \sum_{\Omega^{\prime}} \sqrt{P(I(\mathbf{x})) P\left(I^{\prime}(\mathbf{x}+u(\mathbf{x}))\right)}
$$

In (2), $I(\mathbf{x})$ and $I^{\prime}(\mathbf{x})$ are the previous and current intensity images for which the flow needs to be calculated, $\varphi\left(I(\mathbf{x}), I^{\prime}(\mathbf{y})\right)$ is the ratio of overlapping area to total area between the ellipses representing points $\mathbf{x}$ and $\mathbf{y}$ in the previous and current frames, and the summation term is the Bhattacharyya coefficient of the channel intensities distributions in the two patches $\Omega$ '. The penalty function is bounded between 0 and 1 .

The overlapping area between two generic non-axis aligned ellipses can be calculated first by finding their intersections: two polynomials share a common root if and only if their Bezout determinant is zero, which in the elliptical case is a quartic whose zeros can be efficiently found with a polynomial solver. Given a set of intersections $\Phi$, the area $A_{\text {int }}$ can be found by integrating anticlockwise along the elliptical arcs between intersection points according to Green's theorem:

$$
A_{\mathrm{nth}}=\frac{1}{2} \sum_{\varphi_{i} \in \Phi} R\left(-x_{c} \sin (\theta)+y_{c} \cos (\theta)-\left.\cos (t)\right|_{\varphi_{i}} ^{\varphi_{i+1}}\right)+r\left(x_{c} \cos (\theta)+y_{c} \sin (\theta)+\left.\sin (t)\right|_{\varphi_{i}} ^{\varphi_{i+1}}\right)+\left.R r t\right|_{\varphi_{i}} ^{\varphi_{i+1}}
$$


In Eq. (3), $R, r$ and $\theta$ are the major radius, minor radius and orientation of the ellipse whose arc two consecutive intersections lie on. The integrating variable $t$ is the angle between the ellipse centre and a point. The overlapping area to total area ratio is then:

$$
\varphi(\mathbf{x}, \mathbf{y})=\frac{A_{\text {int }}}{\pi\left(R_{\mathbf{x}} r_{\mathbf{x}}+R_{\mathbf{y}} r_{\mathbf{y}}\right)}
$$

\subsection{Variational Optimisation}

The problem formulated by Eq. (1) can be solved with differentiable data terms in the case where $\psi$ is the TV- $\mathrm{L}_{1}$ operator [8]. The general principle can be extended to cases when non-differentiable penalty functions are used [9]. Here we further extend the method to irregular graphical structures with an arbitrary degree of connectivity. As Eq. (1) is nonconvex in $u$, an auxiliary vector field $v$ is introduced to decouple the pointwise penalty term from the regularity term, leading to the convex approximation:

$$
\min _{u, v}\left\{\int_{\Omega} \lambda g(\mathbf{x}, v(\mathbf{x}))+\frac{1}{2 \theta}(v(\mathbf{x})-u(\mathbf{x}))^{2}+\psi(\nabla u(\mathbf{x})) d \Omega\right\}
$$

Such an approximation is convex in $u$ and nonconvex in $v$, and it can be shown that $u \rightarrow v$ as $\theta \rightarrow 0$. Its solution involves alternating two optimisation steps while decreasing $\theta$ between iterations: for non-differentiable data terms, the optimal $v$ (with constant $u$ ) is found by a complete search. For each iteration $i$, the optimal element of $u$ (with constant $v$ ) for each dimension $d$ can be found by the following scheme:

$$
\left\{\begin{array}{l}
u_{d}^{i+1}=v_{d}^{i+1}-\theta \operatorname{div} p_{d}^{i} \\
p_{d}^{i+1}=\frac{p_{d}^{i}+\tau \nabla\left(\operatorname{div} p_{d}^{i}-v / \theta\right)}{1+\left|\tau \nabla\left(\operatorname{div} p_{d}^{i}-v / \theta\right)\right|}=\frac{p_{d}^{i}+\tau \nabla u_{d}^{i+1}}{1+\left|\tau \nabla u_{d}^{i+1}\right|}
\end{array}\right.
$$

Above, $p$ is a collection of $d k$-dimensional auxiliary vectors each initialised at $p^{0}=0$. The irregular element spacing throughout the graphical model is reflected by the gradient operator which weighs the variation of neighbouring elements proportionally to the reciprocal of their Euclidean $L_{d}$ norm, constraining closer elements more than farther away ones.

\subsection{Applications to MIS}

The above theoretical framework is applied to stereo MIS video for the specific case of $d=3$ and $k=1$. This corresponds to an arbitrary connected trace (open or closed) in 3D space, and can be applied to, for example, tracking custom freehand inputs overlaid on the visualised 3D scene for preoperative path-planning or surgical training. Such interfaces have been investigated for natural scenes [10] with the additional requirement of prior offline processing, and for telementoring in MIS [11] using conventional algorithms on static scenes. The proposed algorithm allows using directly prior $3 \mathrm{D}$ velocity information to restrict the search space for the optimal motion vector and it adds robustness to the process due to the penalty term tailored to 
MIS data. Also, it yields subpixel accuracy if a fine discretisation of the 3D space is adopted. More importantly, contrarily to unconstrained point tracking it strives to preserve the shape of the original annotation due to its regularisation term.

Under this scheme, the user is prompted to draw an annotation on a still frame from one of the stereo channels. Stereo matches for the annotation points are found on the remaining channel with the algorithm from [12], the matching pairs are then triangulated yielding a $3 \mathrm{D}$ representation of the original annotation. The annotation is then tracked across the incoming frames with the algorithm presented. The penalty function is modified to be an average of the two penalties from the projection of the 3D candidate point on the left and right channel and their comparisons with the previous frames, thus enforcing inter-channel consistency. Fig. 2 below schematically illustrates the proposed technique.
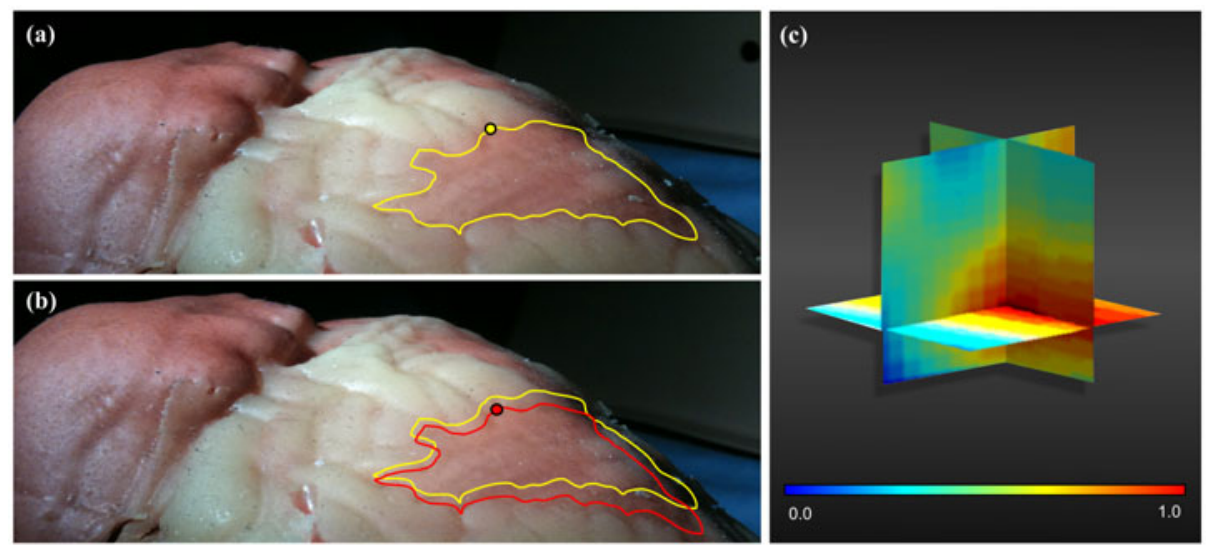

Fig. 2. An illustration of the proposed method. A 3D point (a) can be moved to a new position (b) within a rectangular parallelepipedal window depending on the correlation expressed by the penalty function (c). Uneven displacement between connected points results in stronger constraints from the regularisation term.

\section{Experimental Setup and Results}

The proposed technique has been tested both with a synthetic phantom model and in vivo to evaluate its performance. Both sequences were recorded in standard definition at $25 \mathrm{fps}$ from a da Vinci surgical platform with known stereo camera parameters, and input to an Intel Core 2 Duo $2.4 \mathrm{GHz}$ system with $2 \mathrm{~GB}$ of RAM for processing. With the above setup, the algorithm operates at $\sim 1.5 \mathrm{fps}$. For all sequences, the algorithm considers a velocity search space of $2 \mathrm{~mm}^{3}$ per point per frame sampled at intervals of $0.025 \mathrm{~mm}$, with values for $\lambda$ and $\tau$ set to 1.1 and 0.8 respectively.

Phantom experiment. The method was first applied to the dynamic sequence of a silicone phantom heart (Chamberlain Group, MA, USA) beating at 90bpm. A closed contour with 39 control points was defined in correspondence of a section of the left anterior descending artery (LAD). Fig. 3 (a) and (b) below show the reconstructed annotation volume over a section of the sequence with highlighted diastolic frames. 
The tracked annotation shows a small drift of its left and bottom segment, while its centroid remains focused on the region of interest. The upper and right sections remain stable throughout. The drift is due to the fact that the annotation is crossing two completely uniform areas without feature information. The regularisation constraint however limits the amount of drift by driving the whole volume in a uniform direction determined by the annotation segments crossing feature points. Tracking regularity over the whole sequence is quantitatively shown in Fig. 3 (c):
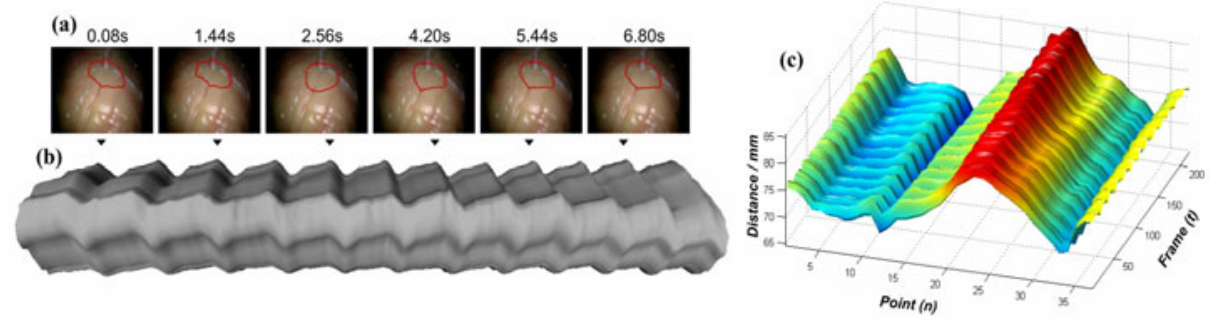

Fig. 3. (a) Selected diastolic frames with the tracked annotation corresponding to ridges in (b). (b) Reconstructed annotation volume over time. (c) Distance of annotation points from the camera over the sequence.

It can be seen that the overall drift is less than $2 \mathrm{~mm}$, while it stabilises in the latter half of the sequence. All other points are stable throughout. The closed-loop configuration is maintained during the sequence, as shown by the surface's sinusoidal characteristics, and alternating cardiac phases for all points are evidenced by the ridges in the plot.

In vivo experiment. The algorithm was further evaluated with a TECAB sequence by delineating an open segment on the pericardium. The sequence presents some significant deformation, with tissue sections becoming occluded from the heart contractions. Fig. 4 below shows the performance of the technique together with selected frames:
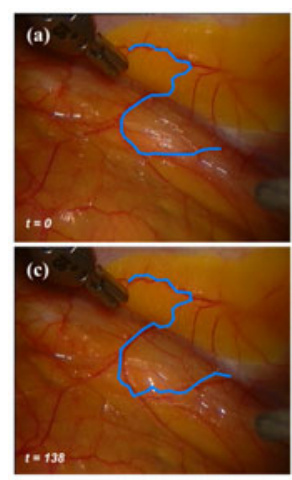
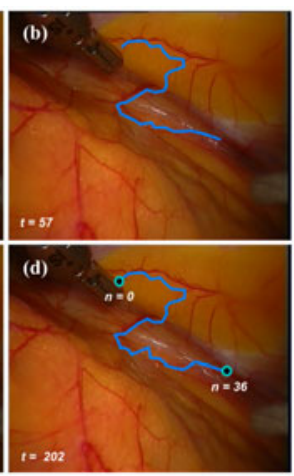

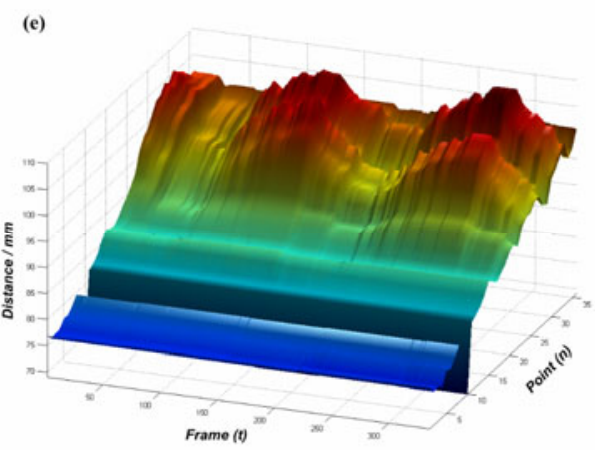

Fig. 4. (a) Initial contour. (b) First diastole. (c) First systole. (d) Second diastole. (e) Distance from camera centre of the tracked points along the annotation over time. 
Despite the degree of tissue deformation shown in Fig. 4 (b), (d) and the lack of explicit occlusion handling, the proposed method was able to maintain the annotation regularity and recover the tissue motion as shown in Fig. 4 (e), where the diastoles and systoles corresponds to the troughs and peaks in the surface plot respectively.

Furthermore, the top half of the images containing the first 15 points of the annotation was motionless throughout the sequence. This is reflected by the surface plot in Fig. 4 (e), where motion characteristics are clearly discernible between the first and last portion of the annotation.

Validation results. To facilitate validation, the phantom heart was augmented with 15 silicone fiducials for registration visible on its surface and scanned with a Siemens Somatom Sensation 64 CT scanner. The resulting volumes had a spatial resolution of $0.41 \times 0.41 \times 0.5 \mathrm{~mm}$ and a temporal resolution of $0.33 \mathrm{~s}$. During the scan the heart rate was set to $90 \mathrm{bpm}$, yielding twenty $3 \mathrm{D}$ volumes covering the entire cardiac cycle.

The cardiac volumes were then manually registered with the video sequence, so that the correspondence between frame number and cardiac phase was known. For quantitative validation, two annotations have been considered: the open contour shown in the phantom experiment section, and a closed contour delineating the LAD. For both sequences, the initial 3D annotation was stored internally together with its corresponding cardiac phase; the distance between the initial 3D points and the estimated annotation position was then computed whenever the frame under consideration mapped to the initial cardiac phase. Table 1 below quantifies the overall performance of the algorithm:

Table 1. Numerical evaluation of the performance from the proposed algorithm

\begin{tabular}{lcccc}
\hline Error statistics $(\mathrm{mm})$ & Mean & $\begin{array}{c}\text { Standard } \\
\text { deviation }\end{array}$ & Maximum & Minimum \\
\hline Open contour & 2.133 & 0.810 & 3.695 & 0.912 \\
Closed contour & 1.792 & 0.721 & 3.561 & 0.433 \\
\hline
\end{tabular}

Overall, the open and closed contour sequences show similar performance. The relatively small error in the case of closed contours is due to stronger constraints imposed. Also, given two segments of equal lengths the closed segment would cover a more localised area of the heart surface which could exhibit a higher degree of motion cross-correlation.

\section{Discussion and Conclusions}

In this paper, we have presented a novel technique for 3D tracking of arbitrary structures with applications to annotation tracking in MIS. Robustness is provided by a novel penalty function while motion consistency is enforced by a TV- $\mathrm{L}_{1}$ regularisation constraint. Tracking directly in 3D space enforces inter-channel consistency and allows for the reduction of the search space with prior motion information without explicit motion models. The technique has been tested on phantom and in vivo with an average discrepancy during validation of $1.79 \mathrm{~mm}( \pm 0.72 \mathrm{~mm})$. The regularity of the algorithm 
during phantom experiments shows its validity, and future work will focus on a realtime implementation exploiting the method's suitability for parallel processing, a reinitialisation procedure for cyclic motion and occlusion detection.

\section{References}

1. Stoyanov, D., Mylonas, G., Yang, G.-Z.: Gaze-Contingent 3D Control for Focused Energy Ablation in Robotic Assisted Surgery. In: Metaxas, D., Axel, L., Fichtinger, G., Székely, G. (eds.) MICCAI 2008, Part II. LNCS, vol. 5242, pp. 347-355. Springer, Heidelberg (2008)

2. Mylonas, G., Kwok, K.W., Darzi, A., Yang, G.-Z.: Gaze-Contingent Motor Channelling and Haptic Constraints for Minimally Invasive Robotic Surgery. In: Metaxas, D., Axel, L., Fichtinger, G., Székely, G. (eds.) MICCAI 2008, Part II. LNCS, vol. 5242, pp. 676-683. Springer, Heidelberg (2008)

3. Mountney, P., Stoyanov, D., Yang, G.Z.: Soft Tissue Tracking for Minimally Invasive Surgery: Learning Local Deformation Online. In: Metaxas, D., Axel, L., Fichtinger, G., Székely, G. (eds.) MICCAI 2008, Part II. LNCS, vol. 5242, pp. 364-372. Springer, Heidelberg (2008)

4. Horn, B.K.P., Schunck, B.G.: Determining optical flow. Artificial Intelligence 17, 18 (1981)

5. Zach, C., Pock, T., Bischof, H.: A Duality Based Approach for Realtime TV-L1 Optical Flow. In: Hamprecht, F.A., Schnörr, C., Jähne, B. (eds.) DAGM 2007. LNCS, vol. 4713, pp. 214-223. Springer, Heidelberg (2007)

6. Steinbruecker, F., Pock, T., Cremers, D.: Advanced Data Terms for Variational Optic Flow Estimation. In: Vision, Modeling, and Visualization Workshop, Braunscweig, Germany (2009)

7. Giannarou, S., Visentini-Scarzanella, M., Guang-Zhong, Y.: Affine-invariant anisotropic detector for soft tissue tracking in minimally invasive surgery. In: IEEE International Symposium on Biomedical Imaging: From Nano to Macro, ISBI 2009, pp. 1059-1062 (2009)

8. Chambolle, A.: An algorithm for total variation minimization and applications. Journal of Mathematical Imaging and Vision 20(1-2), 89-97 (2004)

9. Steinbruecker, F., Pock, T., Cremers, D.: Large Displacement Optical Flow Computation Without Warping. In: IEEE International Conference on Computer Vision, Kyoto, Japan (2009)

10. Rav-Acha, A., Kohli, P., Rother, C., Fitzgibbon, A.: Unwrap Mosaics: A new representation for video editing. In: ACM Transactions on Graphics (SIGGRAPH 2008) (2008)

11. Ali, M.R., et al.: 3-D telestration: a teaching tool for robotic surgery. J. Laparoendosc. Adv. Surg. Tech. 18(1), 107-112 (2008)

12. Stoyanov, D., Darzi, A., Yang, G.Z.: Dense 3D Depth Recovery for Soft Tissue Deformation During Robotically Assisted Laparoscopic Surgery. In: Barillot, C., Haynor, D.R., Hellier, P. (eds.) MICCAI 2004. LNCS, vol. 3217, pp. 41-48. Springer, Heidelberg (2004) 